\title{
Pressure-assisted forming of non-concentric tubular cross sections with solid medium
}

\author{
Alves, Luis M.; Silva, Carlos M.A. ; Nielsen, Chris Valentin; Martins, Paulo A.F.
}

Published in:

Proceedings of the Institution of Mechanical Engineers, Part B: Journal of Engineering Manufacture

Link to article, DOI:

10.1177/0954405415625913

Publication date:

2017

Document Version

Peer reviewed version

Link back to DTU Orbit

Citation (APA):

Alves, L. M., Silva, C. M. A., Nielsen, C. V., \& Martins, P. A. F. (2017). Pressure-assisted forming of nonconcentric tubular cross sections with solid medium. Proceedings of the Institution of Mechanical Engineers, Part $B$ : Journal of Engineering Manufacture, 23(12), 2123-2132. https://doi.org/10.1177/0954405415625913

\section{General rights}

Copyright and moral rights for the publications made accessible in the public portal are retained by the authors and/or other copyright owners and it is a condition of accessing publications that users recognise and abide by the legal requirements associated with these rights.

- Users may download and print one copy of any publication from the public portal for the purpose of private study or research.

- You may not further distribute the material or use it for any profit-making activity or commercial gain

- You may freely distribute the URL identifying the publication in the public portal 


\title{
PRESSURE-ASSISTED FORMING OF NON-CONCENTRIC TUBULAR CROSS SECTIONS WITH SOLID MEDIUM
}

\author{
L.M. Alves ${ }^{a}$, C.M.A. Silva ${ }^{a}$, C.V. Nielsen ${ }^{b}$, and P.A.F. Martins ${ }^{a^{*}}$ \\ ${ }^{a}$ IDMEC, Instituto Superior Técnico, Universidade de Lisboa, Av. Rovisco Pais, 1049-001 Lisboa, \\ Portugal \\ ${ }^{b}$ Department of Mechanical Engineering, Technical University of Denmark, Produktionstorvet 425, 2800 \\ Kgs. Lyngby, Denmark
}

$(*)$ Corresponding author. E-mail: pmartins@ist.utl.pt First author. E-mail: luisalves@ist.utl.pt Second author. E-mail: carlos.alves.silva@ist.utl.pt Third author. E-mail: cvni@mek.dtu.dk 


\section{ABSTRACT}

Pressure-assisted forming of tubes allows producing a wide variety of tubular components that are difficult or impossible to fabricate by means of conventional tube forming. In contrast to previous investigations in the field that were almost exclusively focused on the utilization of fluids (tube hydroforming) or elastomers (tube rubber forming) as pressuring medium, the subject matter of this paper is centred in the utilization of low melting point, recyclable, metallic alloys as solid pressurizing medium.

The aims and scope of the paper are centred on the feasibility of forming straight carbon steel tubes into complex gooseneck geometries with non-concentric cross sections by using lead as a solid pressuring medium and employing a double-action cam driven tool system. The presentation is focused on the tool system, on its adequacy to produce customized tubular components, on the required forming forces and on the typical modes of deformation that result from the different movements provided by the vertical and horizontal actuators of the double-action tool system.

Results and observations confirm that the utilization of a double-action tool system with a solid pressurizing medium to assist plastic deformation and prevent collapse can be successfully and effectively employed to fabricate non-concentric tubular cross sections for prototypes and small batches of lightweight components.

Keywords: Forming, Tubes, Solid pressurizing medium, Double-action tool, Experimentation, Finite element method 


\section{INTRODUCTION}

Cold forming of tubes with simultaneous action of external mechanical load and internal pressure established as an alternative to conventional manufacturing processes based on stamping, welding and casting in the late 1960's and 1970's. The pioneer investigations by Ogura $^{1}$ and by Al-Qureshi ${ }^{2}$ showed that both fluids and elastomers could be successfully utilized as pressurizing medium in place of a hard tool to plastically deform a straight tube into a tee branch tubular component.

From 1960's to nowadays, the utilization of pressurized fluids evolved into tube hydroforming (THF), which is a technology widely utilized to produce large batches of tubular components with rotationally symmetrical (Figure 1a), rotationally unsymmetrical (Figure 1b) and eccentric (or gooseneck) (Figure 1c) cross sections for automotive, household appliances and lightweight structures ${ }^{3}$.



(a)

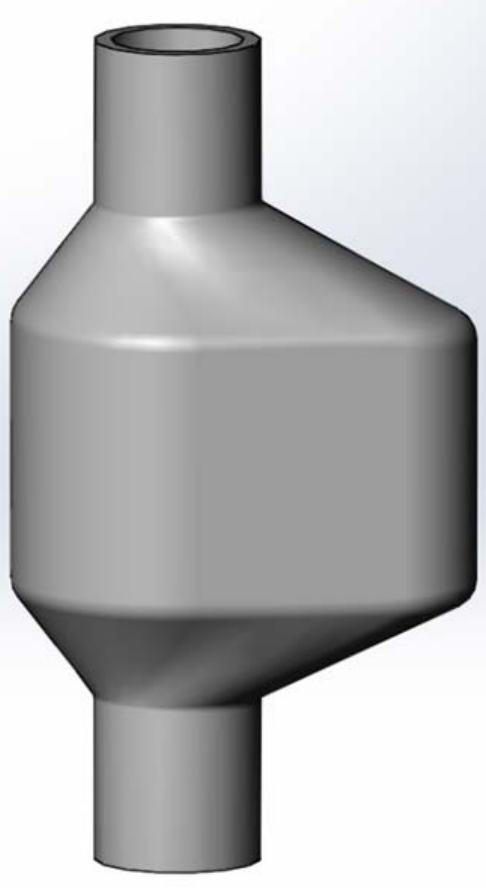

(b)

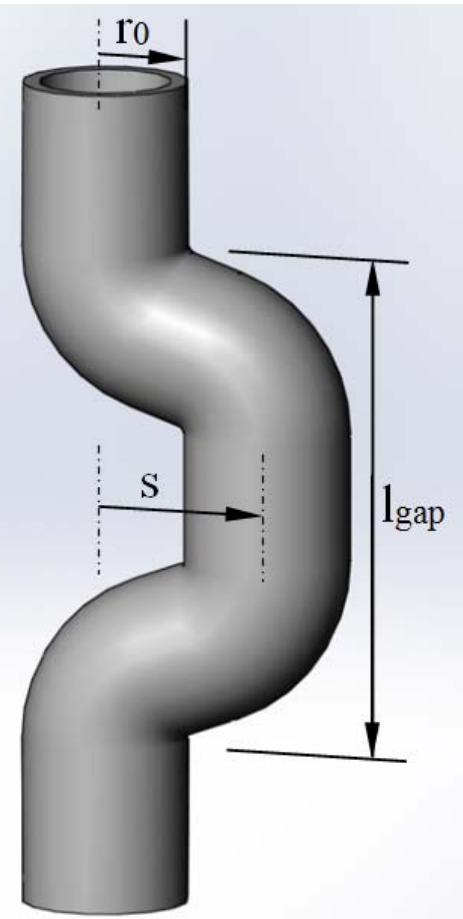

(c)

Figure 1 - Classification of tube hydroforming as a function of the position of the cross section of the plastically deformed regions to the longitudinal axis of the original straight tube: (a) rotationally symmetrical, (b) rotationally unsymmetrical and (c) eccentric (or gooseneck) cross sections. 
The main scientific and technological developments in THF are comprehensively described in the state-of-the-art review performed by Ngaile $^{4}$ who identified the main process parameters as the tubular material, the starting geometry (thickness and length), the desired shape and the loading path; namely the combination of internal pressure and the external axial feeding that are needed to ensure large deformations without failure. A recent publication by Lee et al. ${ }^{5}$ extended the general principles and processes that were covered in the above mentioned publication with recent technological developments and presented new modelling and design strategies for improving formability in THF. The development of pulsed $\mathrm{THF}^{6}$, the understanding of the mechanisms behind the improvement of formability in $\mathrm{THF}^{7}$, the utilization of advanced high strength steels and magnesium alloys and the utilization of new design and modelling strategies for achieving larger expansion ratios ${ }^{8}$ and estimating the uncertainties in corner die filling ${ }^{9}$ are some examples of the recent advances in THF. Still, the main drawbacks of THF are the large cycle time, the capital investment in machines and tools (inhibiting small batch production), the safety precautions associated with the use of fluids subjected to very high pressures and the design guidelines that prevent the specification of tight bends with very sharp corners.

Similarly to THF, the utilization of elastomers as solid pressurizing mediums evolved from 1960's to nowadays into tube rubber forming (TRF), which is an effective and widespread technology to produce small batches of tubular components with rotationally symmetric (Figure 1a) and rotationally unsymmetrical (Figure 1b) cross sections containing simple counterdrafts and local expansions.

The main scientific and technological developments in TRF are described in the stateof-the-art review performed by Thiruvarudchelvan ${ }^{10}$ and covered in a comprehensively and broader range in the book recently published by Ramezani and Ripin ${ }^{11}$. The main 
advantages of TRF compared to THF are the elimination of the need to use robust hydraulic presses with plenty of daylight for installing the die sets, the elimination of the difficulties that arise from sealing fluids subjected to very high pressures and the aforementioned economic and flexible production benefits. The main drawbacks of TRF are mainly related to the limited amount of displacement that elastomers can successfully withstand, to the necessity of specifying relief gaps in tools in order to prevent excessive compression and damage of the elastomers, and to the difficulties in removing elastomers from complex tubular components with rotationally eccentric (or gooseneck) cross sections (Figure 1c).

The above mentioned drawbacks are the main reason why TRF is mainly applied in the production of tubular components with rotationally symmetrical or tee branched cross sections ${ }^{12}$. Very recently Alves et al. ${ }^{13}$ combined TRF with press working compression beading to surpass some of the above mentioned limitations and successfully produce large-width compression beads in tubes that successfully extend the formability limits of conventional compression beading.

In an effort to reach higher values of internal pressure, Qin and Balendra ${ }^{14}$ proposed the replacement of elastomers by thermoplastics as solid pressurizing medium. Their work demonstrates the feasibility of thermoplastics to assist material flow and prevent collapse by buckling during injection forging of thick-walled tubes, but does not address the problem of its removal from the final shaped components. In fact, the removal of thermoplastics by melting is time consuming and likely to produce toxic gases, and the alternative of using solvents is constrained by environmental issues ${ }^{15}$.

The utilization of low melting point metallic alloys as solid pressurizing medium that can be easily removed after forming was originally proposed by Mac Donald and Hashmi $^{16}$ who performed a finite element investigation on the feasibility of producing a 
cross-branch rotationally unsymmetrical tubular component using lead as a pressurizing medium. Their numerical estimates indicate that lead can be successfully utilized as a pressurized medium to assist plastic deformation and prevent failure and that its utilization should give rise to less thinning and lower applied stresses in the crossbranches when compared to THF. However, no experimental results were performed to validate their conclusions.

In contrast to THF and TRF, pressure-assisted forming with low melting point alloys is not limited by the maximum allowable operating pressure of the hydraulic system or by the maximum allowable deformation that elastomers can withstand. This opens the possibility to form thicker tubes than those commonly produced by THF and TRF.

This paper draws from the abovementioned proposal of using lead as a solid pressurizing medium to the development of a flexible, low cost, pressure-assisted cold forming technology to manufacture low batches of tubular components with eccentric (or gooseneck) cross sections (Figure 1c). Potential applications of these components span from prototypes to custom lightweight structures and cranked tubes. Experiments in a double-action forming tool that was specially designed for the process and threedimensional finite element simulations using an in-house finite element computer program give support to the presentation.

\section{MATERIAL AND METHODS}

This section summarizes the mechanical characterization of the materials, describes the double-action tool system that was designed to produce tubular components with nonconcentric cross sections by pressure-assisted forming with a solid medium, and provides details on the experimental work plan. 


\subsection{Materials}

The raw materials utilized in the investigation consisted of commercial S460MC (carbon steel) welded tubes in the 'as-received' condition and ingots of technically pure lead $(\mathrm{Pb}$ 99.9\%).

The stress-strain curves of the tube materials were determined by means of tensile and stack compression tests carried out at room temperature. The tensile test specimens were machined from the supplied tube stock and the stack compression test specimens were assembled by pilling up circular discs cut from the tube stock by a hole-saw. The stress-strain curve of technically pure lead was determined by means of compression tests carried out at room temperature in specimens machined from the ingots.

The tests were performed on a hydraulic testing machine (Instron SATEC $1200 \mathrm{kN}$ ) with a cross-head speed equal to $100 \mathrm{~mm} / \mathrm{min}(1.7 \mathrm{~mm} / \mathrm{s})$ and the resulting stress-strain curves were approximated by the following Ludwik-Hollomon’s equations,

$$
\begin{array}{rr}
(\mathrm{S} 460 \mathrm{MC}) & \sigma=616 \varepsilon^{0.06}(\mathrm{MPa}) \\
(\mathrm{Pb} 99.9 \%) & \sigma=33.6 \varepsilon^{0.27}(\mathrm{MPa})
\end{array}
$$

The effects of temperature, strain rate and anisotropy were neglected.

\subsection{Double-action tool system}

Friction between the tube and the solid pressurizing medium helps to build up axial compressive stresses on the tube and to eliminate the need to devise means of applying axial feeding independently, as in case of THF. This enables simple tubular components as those shown in Figures 1a and 1b to be produced in tools installed in single-action presses. 
However, tubular components with complex rotationally eccentric (or gooseneck) cross sections (Figure 1c) require multiple actions on the tube in vertical and horizontal directions, individually or in combination to produce the desired shape. This can be provided by custom designed double-action hydraulic presses with two independent sliders or, alternatively, by means of double-action tools installed in single-action presses.

Figure 2 presents a two-dimensional schematic representation of the double-action tool system that was designed and fabricated to produce tubular components with eccentric (gooseneck) cross sections by pressure-assisted forming using lead as a solid medium. The double-action tool system is based on a cam sliding mechanism and consists of structural and process dedicated parts (Figure 2a). The structural parts comprise a plurality of individual parts such as the upper and lower die shoes (UDS, LDS), the guiding columns (GC), the die segments (DS), the die actuator (DA), the sliding die holders (SDH), the guiding lath (GL), the ram holder (RH) and the pre-compressed bars (PCB), which are independent of the geometry of the tubular components with nonconcentric cross sections to be fabricated. The ram (R), the segmented pressure rings (SPR) and the two dies (D) are the process dedicated parts that are dependent on the initial and final geometries of the tubular components to be fabricated. In fact, by changing the ram, the segmented pressure rings and the dies it is possible to produce tubular components with different geometries.

The vertical movement is accomplished through the independent movement of the ram (R) and is used to bend the tube during the initial preforming stage. This type of movement requires the die actuators (DA's) not to get in touch with the sliding die holders (SDH's). 

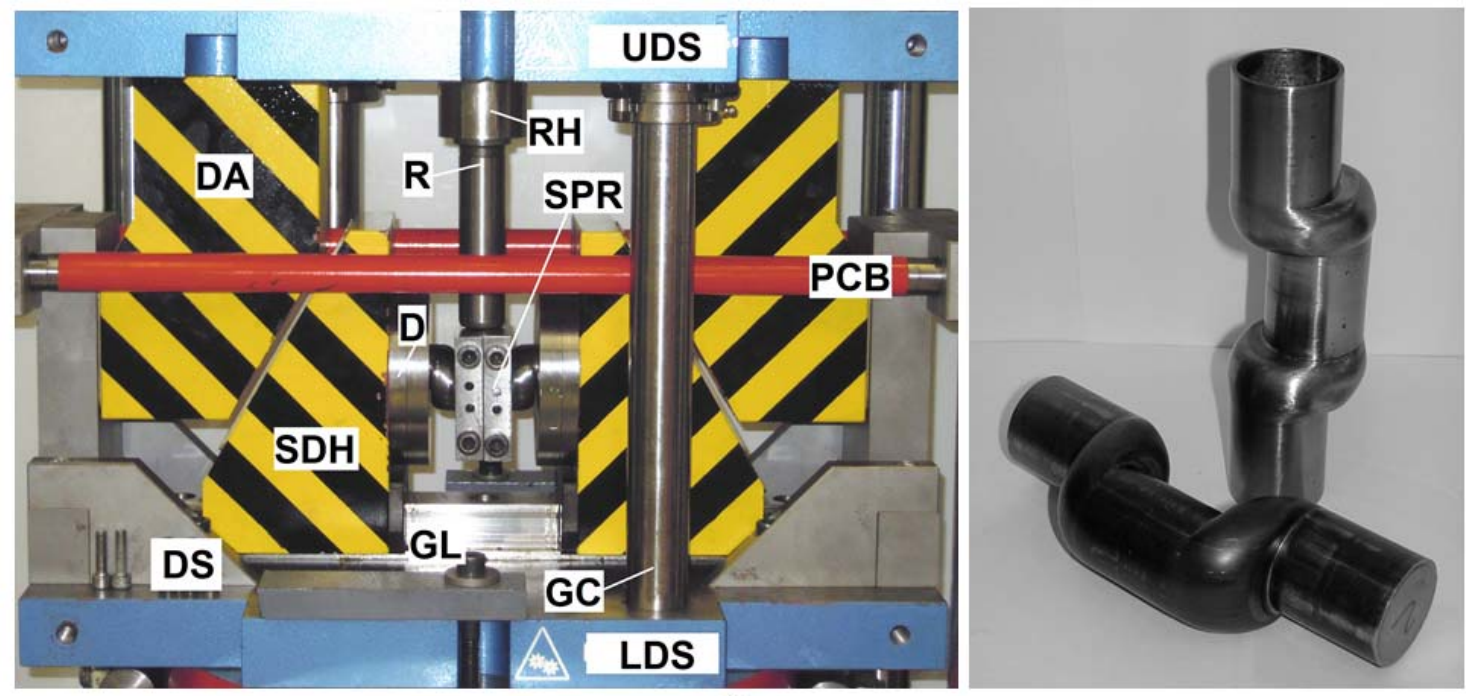

(a)
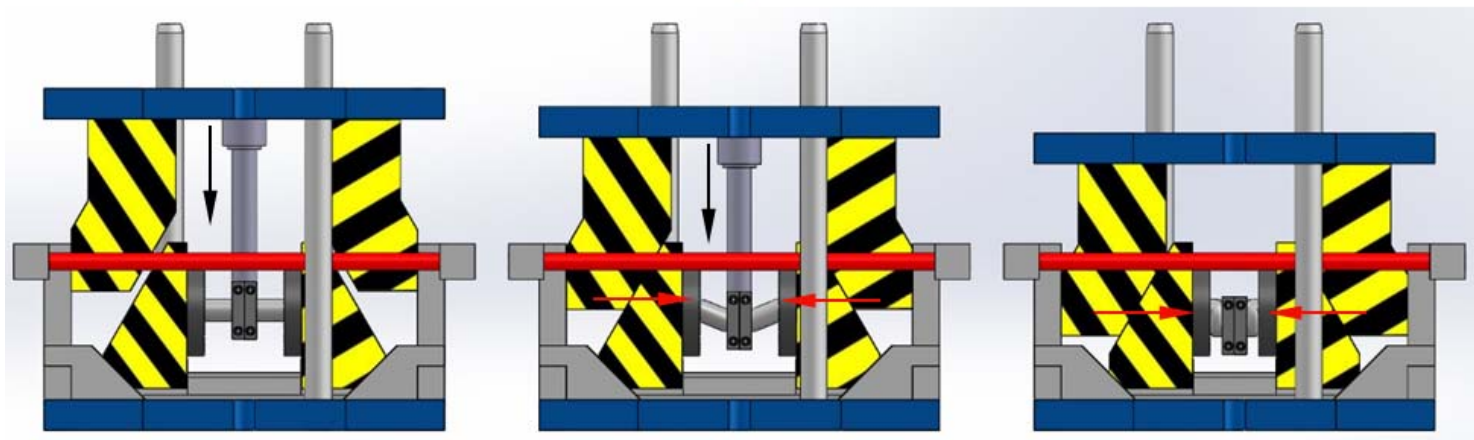

(b)

Figure 2 - Tubular components with eccentric (gooseneck) cross sections produced by pressure-assisted forming with a solid medium.

a) Photograph of the double-action flexible tool system and tubular components after forming and after removing the solid medium and polishing;

b) Schematic representation of the three movements delivered by the tool system (left vertical movement, middle - combined vertical-horizontal movement and right horizontal movement).

The combined vertical-horizontal movement is performed by simultaneous movement of the ram (R) and the sliding die holders (SDH's) and is employed to shape the side webs of the tubular gooseneck geometry by means of controlled plastic instability (buckling) under axial compression loading and internal pressure, as will be seen later in the presentation. This type of movement requires the ram (R) to touch the segmented pressure rings (SPR's) and the die actuators (DA's) to touch the sliding die holders (SDH's) in order to transmit the forming force through the mechanical cam system. 
The horizontal movement is accomplished through the independent movement of the sliding die holders (SDH's) and is used to finish forming the tubular part by plastic instability (buckling). This type of motion requires the ram (R) not to get in touch with the segmented pressure rings (SPR's).

\subsection{Experimental work plan}

The experiments were carried out in the double action tool system installed in the $1200 \mathrm{kN}$ hydraulic testing machine where the mechanical characterization of the materials had previously been performed.

Straight tubular specimens of commercial S460MC carbon steel tubes with an outer radius $r_{0}=16 \mathrm{~mm}$ and a wall thickness $t_{0}=1.5 \mathrm{~mm}$ were prepared from the supplied raw material so as to allow the plan of experiments listed in Table 1 . The work plan was designed in order to deal with three of the most important parameters that were identified during the development of the manufacturing process:

(a) the different movements provided by the actuators of the double-action tool system;

(b) the limiting amount of displacement $s$ in the direction perpendicular to the longitudinal axis of the tube;

(c) the initial unsupported length $l_{\text {gap }}$ of the straight tubular specimens. 


\begin{tabular}{|c|c|c|c|c|c|c|c|c|}
\hline $\begin{array}{l}\text { Test } \\
\text { case }\end{array}$ & $\begin{array}{c}l_{0} \\
(\mathbf{m m})\end{array}$ & $\begin{array}{c}l_{g a p} \\
(\mathbf{m m})\end{array}$ & $\begin{array}{c}r_{0} \\
(\mathbf{m m})\end{array}$ & $\begin{array}{c}t_{0} \\
(\mathbf{m m})\end{array}$ & $\begin{array}{l}\text { Solid pressurizing } \\
\text { medium }\end{array}$ & $\begin{array}{c}\text { Vertical } \\
\text { movement } \\
(\mathrm{mm})\end{array}$ & $\begin{array}{c}\text { Vertical-horizontal } \\
\text { movement } \\
(\mathrm{mm})\end{array}$ & $\begin{array}{c}\text { Horizontal } \\
\text { movement } \\
(\mathrm{mm})\end{array}$ \\
\hline 1 & \multirow{6}{*}{170} & \multirow{6}{*}{98} & \multirow{9}{*}{16} & \multirow{9}{*}{1.5} & No & 1.5 & $6.5-7.5$ & 23.1 \\
\hline 2 & & & & & Yes & 25 & & \\
\hline 3 & & & & & Yes & & & 32.3 \\
\hline 4 & & & & & Yes & 7 & & 24.2 \\
\hline 5 & & & & & Yes & & $25-28.8$ & \\
\hline 6 & & & & & Yes & 7 & $8-9.2$ & 26.6 \\
\hline 7 & \multirow{3}{*}{190} & \multirow{3}{*}{118} & & & Yes & & $25-28.8$ & \\
\hline 8 & & & & & Yes & & $42-48.5$ & \\
\hline 9 & & & & & Yes & 7 & $25-28.8$ & 8.1 \\
\hline
\end{tabular}

Table 1 - The experimental work plan (terminology in accordance to Figure 1).

The investigation on the different movements of the actuators is very important because of their influence on the overall performance and feasibility of the process, and on the total time required to produce a tubular component. In particular, the movement of the vertical actuator plays a key role in setting up the limiting amount of displacement $s$ in the direction perpendicular to the longitudinal axis of the tube that an eccentric (gooseneck) tubular component is capable of withstanding without failure.

The initial unsupported length $l_{\text {gap }}$ of the straight tubular specimen filled with lead as a solid medium is also very important because the slenderness ratio $l_{g a p} / r_{0}$ of the initial unsupported length and the radius influences collapse by buckling and determines the overall quality of the component in the transition region from the original to the eccentric, displaced, cross section.

The wall thickness $t_{0}$ of the tubular specimens was kept at constant value in order to reduce the overall number of parameters that influence the feasibility window of the process.

Lead was cast into cylindrical mandrels that were subsequently installed inside the straight tubular specimens, prior to forming, in order to assist plastic deformation and provide internal pressure to prevent collapse by buckling. The manufacturing process took advantage of material recyclability and mould reutilization because after forming 
the desired geometry of the tubular components, the mandrels were removed by heating and melting at a temperature of approximately $300^{\circ} \mathrm{C}$, while leaving the tubular components intact and ready for subsequent cleaning and polishing.

In connection to this, it is important to state that the proposed technology is not limited to the utilization of lead and its alloys, which require removal by melting to be always performed in well ventilated areas with good exhaustion of fumes in order to avoid poisoning by lead oxide. In fact, the only requirement of the proposed technology is the utilization of ductile, recyclable, low melting point alloys and, therefore, alternative low melting point alloys made from bismuth and tin can easily replace lead with benefits for both environment and health.

The tubular specimens corresponding to Case 1 of Table 1 were tested without pressurizing medium (without internal mandrel) and included in the experimental work plan for reference purposes. 


\section{FINITE ELEMENT MODELLING}

The manufacture of tubular components with non-concentric cross sections by pressureassisted forming with a solid lead medium was performed under a quasi-static constant displacement rate of the upper die shoe $(100 \mathrm{~mm} / \mathrm{min})$. Under these conditions, no inertial effects on plastic deformation are likely to occur and therefore no dynamic effects needed to be considered.

These operating conditions allowed numerical modelling of material flow produced by plastic deformation to be performed with the finite element flow formulation and enabled the authors to utilize the in-house computer program I-form that has been extensively validated against experimental measurements of metal forming processes since the end of the 1980 's ${ }^{17}$.

The finite element flow formulation giving support to I-form is built upon the following extended variational statement accounting for contact and friction between different rigid and deformable objects:

$$
\begin{aligned}
\Pi & =\int_{V} \bar{\sigma} \dot{\bar{\varepsilon}} d V+\frac{1}{2} K \int_{V} \dot{\varepsilon}_{v}^{2} d V-\int_{S_{T}} T_{i} u_{i} d S+\int_{S_{f}}\left(\int_{0}^{\left|u_{r}\right|} \tau_{f} d u_{r}\right) d S \\
& +\frac{1}{2} P \sum_{c=1}^{N_{c}}\left(g_{n}^{c}\right)^{2}+\frac{1}{2} P \sum_{c=1}^{N_{c}}\left(g_{t}^{c}\right)^{2}
\end{aligned}
$$

where $\bar{\sigma}$ denotes the effective stress, $\dot{\bar{\varepsilon}}$ is the effective strain rate, $\dot{\varepsilon}_{V}$ is the volumetric strain rate, $K$ is a large positive constant imposing incompressibility of volume $V, T_{i}$ and $u_{i}$ are the surface tractions and velocities on surface $S_{T}, \tau_{f}$ and $u_{r}$ are the friction shear stress and the relative velocity on the contact interface $S_{f}$ between the tube and the dies or the segmented pressure rings, which were considered as rigid in the numerical simulation. 
The last two terms in (2) account for contact between the counterfacing surfaces of the tube and the solid pressurizing medium. The contact is defined by $N_{c}$ contact pairs that are identified by a two-pass node-to-face algorithm, where the face is the quadrilateral element face of a hexahedral element. Figure 3 shows a schematic contact pair with the surface element divided into four unique triangles by a temporary center node for determining the surface normal in the contact pair.

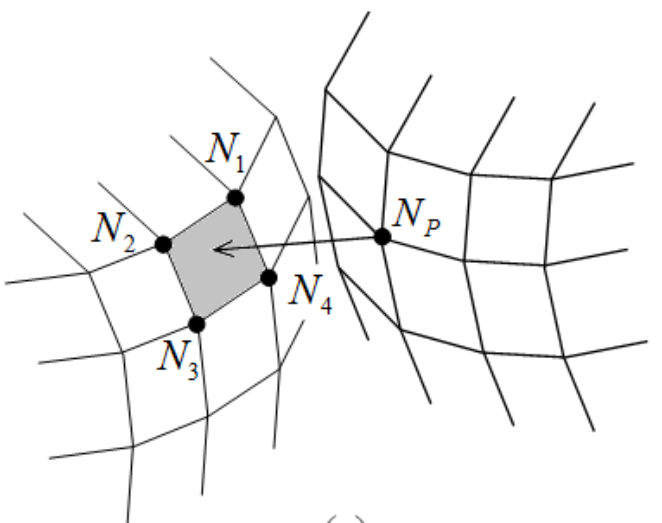

(a)

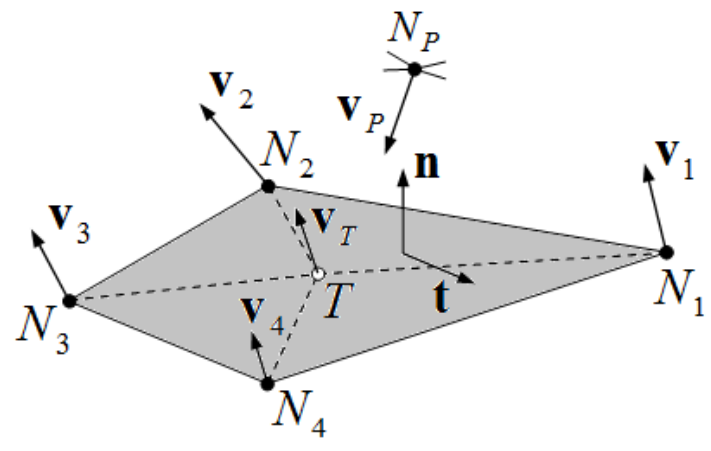

(b)

Figure 3 - Schematic representation of a contact pair in the contact between the tube and the lead pressuring medium and associated notation.

a) Contact pair identified by nodal point $N_{P}$ and opposing quadrilateral element face $N_{1}-N_{2}-N_{3}-N_{4}$

b) Contact pair notation in terms of normal and tangential versors $\mathbf{n}$ and $\mathbf{t}$ in one of the four unique triangular facets appearing by the introduction of a temporary center node $T$. The nodal velocities are denoted $\mathbf{v}$ with the relevant subscript.

The normal gap velocity $g_{n}^{c}$ in the contact pair is identified as,

$$
g_{n}^{c}=\left(\mathbf{v}_{P}-\left(\alpha_{1}+\frac{\alpha_{T}}{4}\right) \mathbf{v}_{1}-\left(\alpha_{2}+\frac{\alpha_{T}}{4}\right) \mathbf{v}_{2}-\frac{\alpha_{T}}{4} \mathbf{v}_{3}-\frac{\alpha_{T}}{4} \mathbf{v}_{4}\right) \cdot \mathbf{n}
$$

and the tangential gap velocity $g_{t}^{c}$ is found similarly by replacing the normal versor $\mathbf{n}$ by the tangential versor $\mathbf{t}$. The normal and tangential versors are defined in Figure 3 together with the nodal velocities appearing in (3). The area coordinates $\alpha_{1}, \alpha_{2}$ and $\alpha_{T}$ 
of the triangle $N_{1}-N_{2}-N_{T}$ are utilized in weighting the nodal velocities for calculating the surface velocity in the nodal projection point of the opposing node. The velocity of the temporary center node is the average of the four element nodes during establishment of (3) due to the linear shape functions.

The normal gap velocity $g_{n}^{c}$ is penalized by a large positive number $P$ in the second last term in (2) when otherwise being negative corresponding to penetration in the contact pair. The last term in (2) is penalizing tangential gap velocity when simulating full sticking. The penalization of tangential gap velocity is omitted when simulating frictionless or frictional sliding, where in the latter case, frictional stresses are applied to the contacting surfaces through the third term in (2) which involves surface tractions. A more detailed description of the frictional sliding is given elsewhere ${ }^{18}$.

On account of symmetry, and because no anisotropy effects due to material or to the welding seam of the carbon steel tubes were taken into consideration, only one quarter of the initial straight tubular specimens and of the solid pressurizing medium needed to be discretized by means of hexahedral finite elements. Three layers of hexahedral finite elements were employed across the tube wall and no remeshing operations were needed to accomplish the final desired shape. The corresponding die and contact surface of the ram with the segmented pressure ring were discretized by means of spatial triangular contact-friction elements as shown in Figure 4a whereas the pressure ring was discretized by a layer of hexahedral elements and modelled as a very stiff (nearly rigid) deformable body. The frictional effects were modelled by means of the law of constant friction with a friction factor $m=0.1$ and the movements of the die and ram were defined in accordance with the values of Table 1.

Figure $4 \mathrm{~b}$ shows half of the real tubular part at the end of the pressure-assisted forming process. The overall CPU time for a typical analysis consisting of a structured mesh 
with approximately 9000 elements was approximately equal to $10 \mathrm{~h}$ on a standard computer equipped with an Intel Xeon E5-2620 (2.10 GHz) processor when using 8 threads.

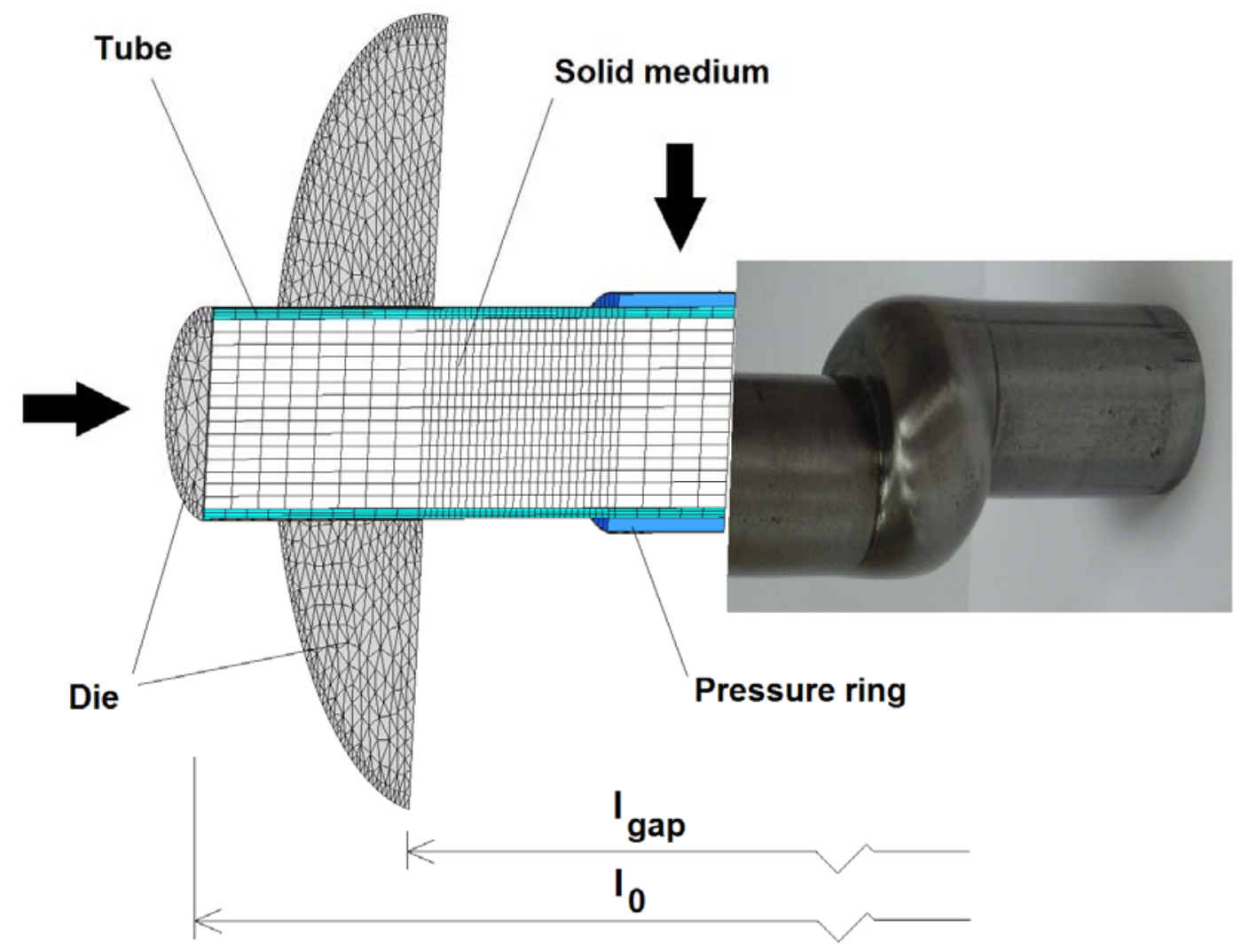

Figure 4 - $\quad$ (a) Finite element model utilized in the numerical simulation of the manufacture of tubular components with eccentric (gooseneck) cross sections by pressure-assisted forming with a solid lead medium.

(b) Real tubular component at the end of the manufacturing process.

\section{RESULTS AND DISCUSSION}

\section{1 - Modes of deformation}

Figure 5 presents the typical modes of deformation that occur during the manufacture of tubular components with eccentric (gooseneck) cross sections by pressure-assisted forming with a solid lead medium in a double-action tool system. 
The mode of deformation of the leftmost tubular component (labelled as 'Case 1' in Figure 5) corresponds to collapse by buckling and reveals significant changes of the cross sectional shape from circular to elliptical due to absence of solid (lead) pressurizing medium. This mode of deformation is not appropriate to produce tubular components with eccentric (gooseneck) cross sections.

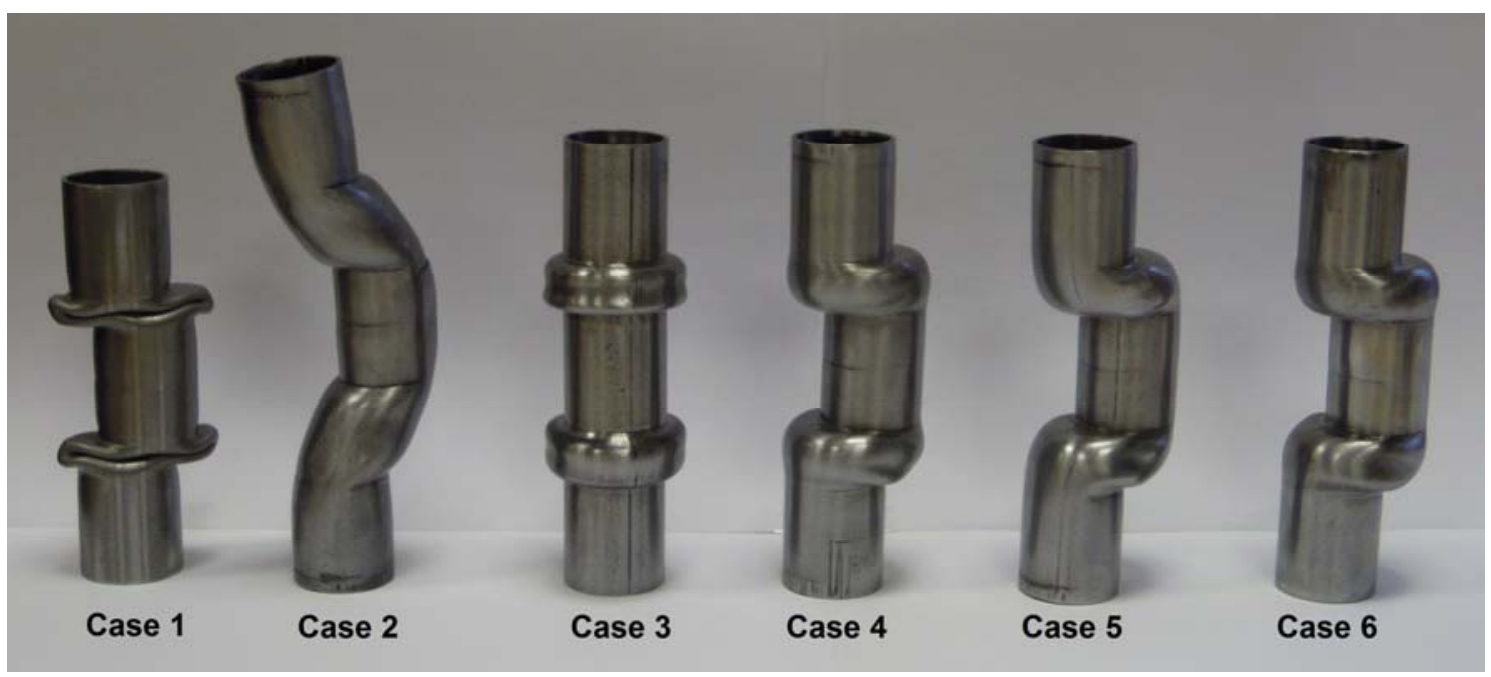

Figure 5 - Tubular components obtained from different movements of the vertical and horizontal actuators of the double-action tool system showing non-appropriate (Cases 1 to 3 of Table 1) and appropriate (Cases 4 to 6 of Table 1) modes of deformation.

The mode of deformation of the following tubular component (labelled as 'Case 2' in Figure 5) corresponds to collapse by fracture in opening mode I (by tension) at the outermost region. This mode of deformation is also not appropriate to produce tubular components with eccentric (gooseneck) cross sections and is caused by an excessive stroke of the vertical actuator (in the direction perpendicular to the longitudinal axis of the straight tubular specimens) that produces a bended preform with inadmissible wall thinning in the outermost region of the tube subject to stretching (tensile elongation). Signs of localized necking can easily be observed in this region of the preforms before fracture.

The mode of deformation of the tubular component labelled as 'Case 3' (Figure 5) is also not appropriate to produce geometries with eccentric (gooseneck) cross sections 
because material flows uniformly around the axis of the original straight tubular specimen to form a concentric double-headed (rotational symmetric, Figure 1a) instead of a gooseneck geometry, as a result of local plastic instability. This result puts into evidence the key role played by bended preforms produced by the vertical actuator (imposing movement in the direction perpendicular to the longitudinal axis of the straight tubular specimens) whenever tubular components with non-concentric cross sections are to be produced.

The three remaining tubular components labelled as 'Case 4', 'Case 5' and 'Case 6' (Figure 5) exhibit eccentric (gooseneck) cross sections but were fabricated with different movements of the horizontal and vertical actuators of the double-action tool system. The specimen labelled as Case 4, for example, was produced by means of two independent movements of the actuators; firstly, the vertical actuator (ram) was forced against the pressure rings in order to produce a bended preform and, secondly, the horizontal actuator (sliding dies) finished shaping the tubular component by means of plastic buckling under axial compression and internal pressure. However, due to limitations on the amount of bending associated with the movement of the vertical actuator (refer to Case 2), this solution is not appropriate for producing tubular gooseneck components with large eccentricity (i.e. with large distance to the centre of the original cross section of the straight tubular specimen).

In contrast to Case 4, the tubular component labelled as Case 5 was fabricated in a single forming stage through combined action of the vertical and horizontal actuators. The main advantage of this solution over the previously mentioned procedure based on single independent actions of the vertical and horizontal actuators is the reduction of the total time required to produce a tubular component with gooseneck geometry. 
Finally, Case 6 was produced by combination of single and multiple actions of the vertical and horizontal actuators. In the first stage, the vertical actuator (ram) forces the pressure rings against the straight tubular specimen to produce a bended preform and, as the upper tool shoe continues to descend, the die actuators get in touch with the sliding die holders and start transmitting the horizontal force that is needed for material undergoing controlled plastic buckling under axial compression and internal pressure. Then, in the second stage, the vertical actuator (ram) is removed and the tubular component is finished by a final amount of plastic buckling imposed by the horizontal action of the sliding dies.

The finite element predicted shapes of the tubular component at an intermediate displacement of the first stage and at the final displacements of the first and second stages are disclosed in Figure 6 for Case 6. The predicted distribution of effective strain $\bar{\varepsilon}$ resulting from finite element analysis allows identifying dead metal zones (DMZ’s) at the edges and central regions of the tubes (and solid lead medium) where the deformation is negligible and zones of high deformation in the transition between the original and the eccentric cross sections of the tubes and solid lead medium due to accumulation of high tension (HT) and high localized shearing (HLS), respectively.

The comparison between Cases 5 and 6 in Figure 5 also reveals that for similar amounts of displacement $s$ in the direction perpendicular to the longitudinal axis of the straight tubular specimen, Case 6 is the best option because the larger curvature of Case 5 at the transition region from the original to the eccentric, displaced, cross sections is a consequence of higher stretching and thinning in the outermost walls.

The photographs enclosed in Figure 7 show how the initial unsupported length $l_{\text {gap }}$ of straight tubular specimens influence the final geometry at the transition regions and help setting up the maximum achievable displacement $s$ in the direction perpendicular to the 
longitudinal axis that a gooseneck tubular component can successfully withstand without failure.

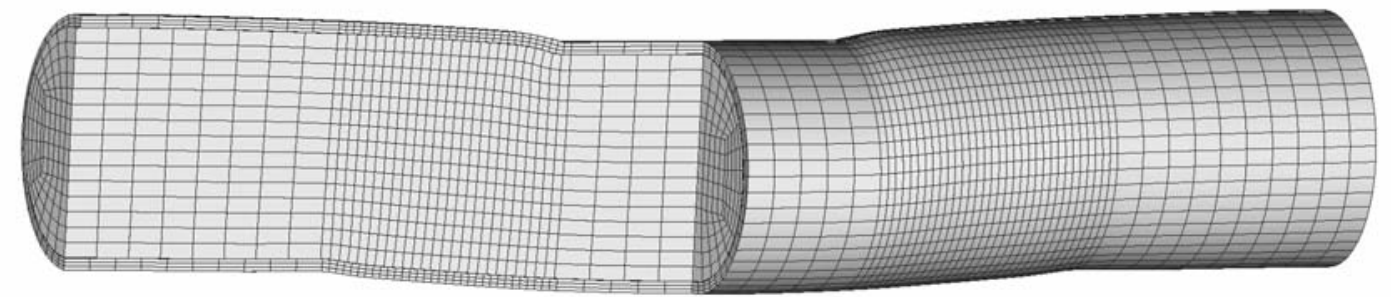

(a)

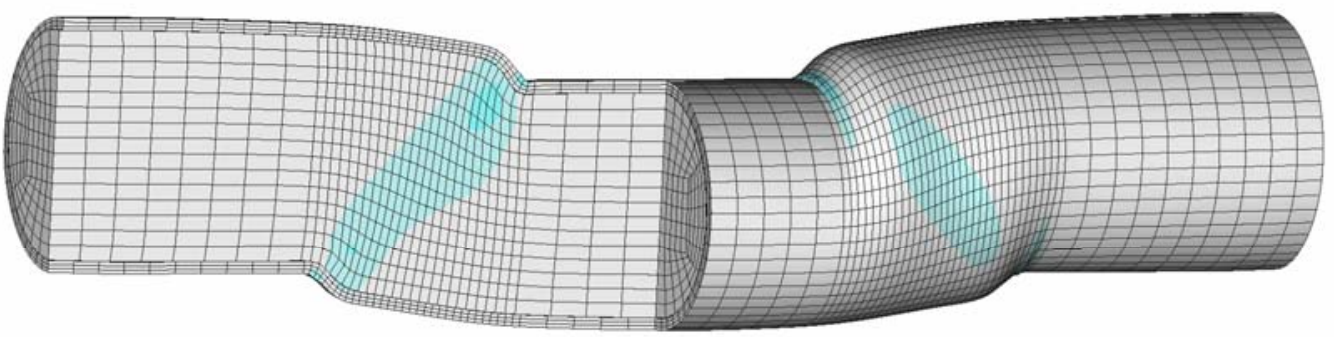

(b)

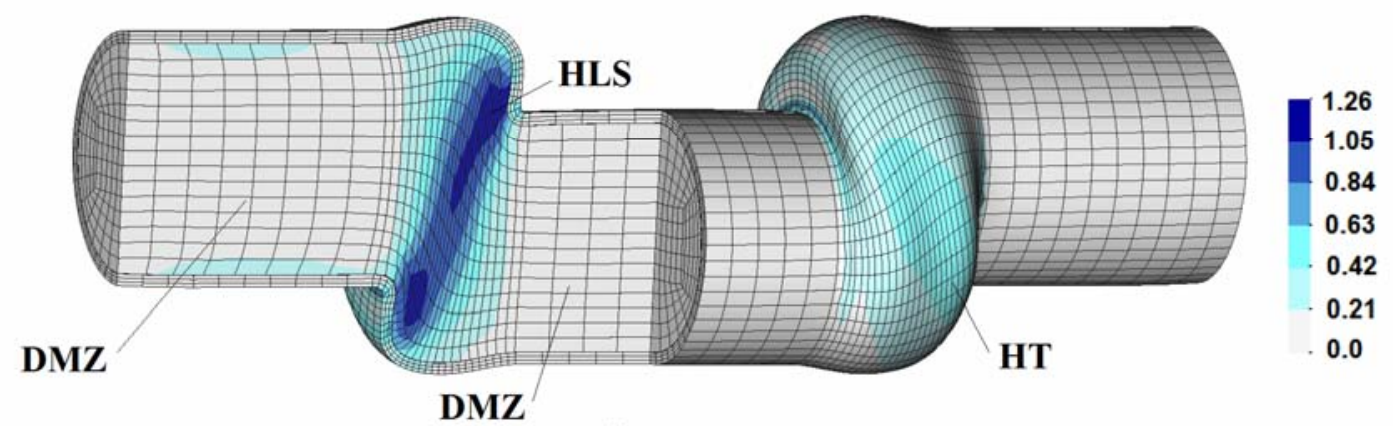

(c)

Figure 6 - Finite element predicted geometry and distribution of effective strain for Case 6 of Table 1 at a displacement of the upper tool shoe corresponding to the (a) end of preform bending, (b) end of the first stage and (c) end of the second and final stage.

As seen in Figure 7a, both tubular components labelled as 'Case 5' and 'Case 7' are capable of withstanding displacements of the vertical actuator of the double-action tool system (in the direction perpendicular to the longitudinal axis of the tube) up to a limiting value $s \cong 2 r_{0}$. However, the geometry of the tubular component with the largest $l_{\text {gap }}$ (that is, Case 7) is more curved and reveals signs of unacceptable wrinkling along the transition from the original to the eccentric, displaced, cross sections. The 
development of wrinkling is attributed to excessive drag and localized lacks of internal pressure by the solid lead medium when the development of very high shearing inside the medium gives rise to confinement of plastic deformation along shear bands.

However, it is worth noting that apart from the extreme forming conditions of Case 7 and in close accordance to TRF, friction between the low melting point alloys and the tube wall is beneficial to form more uniform and wrinkle-free components than those produced by conventional tube hydroforming.

Once the maximum achievable displacement is exceeded $\left(s>2 r_{0}\right)$ thinning of the tube wall becomes inadmissible and specimens collapse by fracture in opening mode I (by tension). This is clearly seen in Case 8 of Figure $7 \mathrm{~b}$, where $s \cong 40 \mathrm{~mm}$ (that is, $\left.S=2.5 r_{0}\right)$.

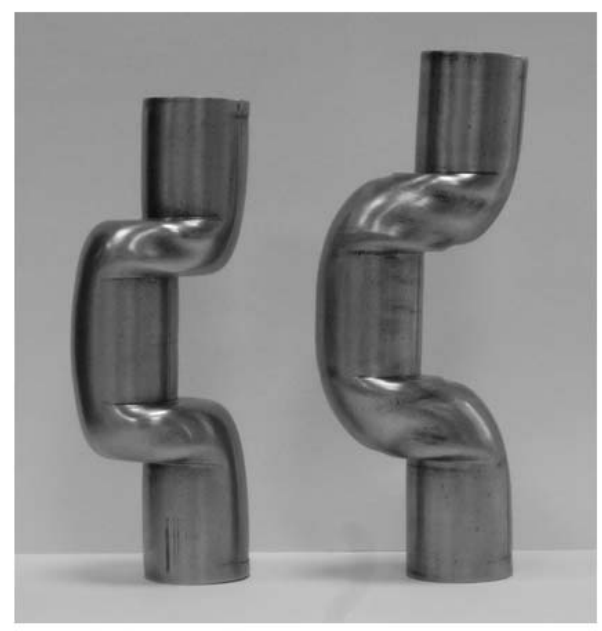

(a)

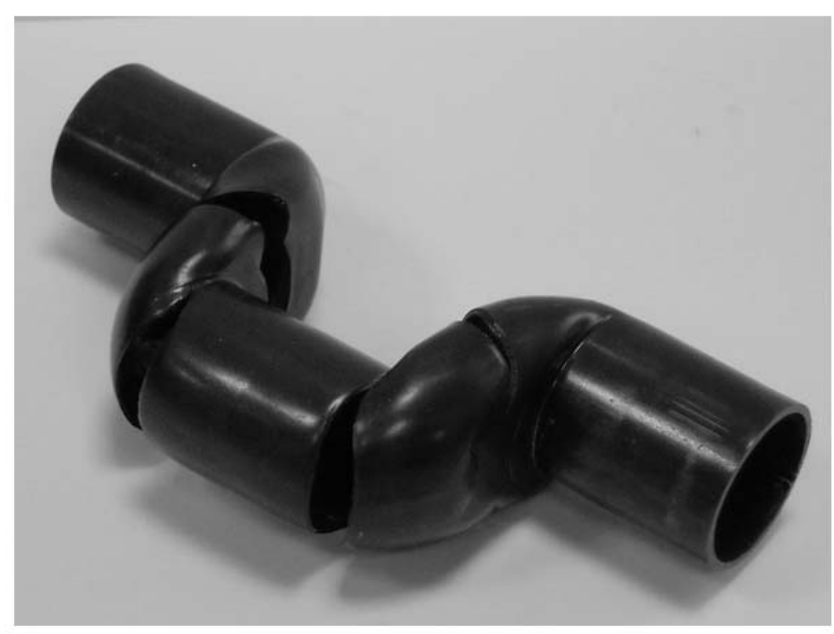

(b)

Figure 7 - Influence of two main process parameters in the overall performance of the process.

a) Photograph of Cases 5 and 7 showing the influence of the initial unsupported length $l_{\text {gap }}$ on the final geometry of the tubular parts ( $S \cong 2 r_{0}$ in both cases).

b) Photograph of Case 8 (without final polishing) showing failure by fracture in opening mode I when the displacement in the direction perpendicular to the longitudinal axis of the straight tubular specimen is excessive ( $S>2 r_{0}$ ). 


\section{2 - Forming forces}

Figure 8 shows the finite element predicted and experimental evolution of the loaddisplacement curve for Case 6 of Table 1. As seen, both evolutions compare well and allow distinguishing three different regions corresponding to the three different movements of the actuators of the double-action tool system; (i) independent movement of the vertical (ram) actuator (labelled as 'A'), (ii) combined movement of the vertical and horizontal actuators (labelled as 'B') and (iii) independent movement of the horizontal (sliding dies) actuators (labelled as 'C').

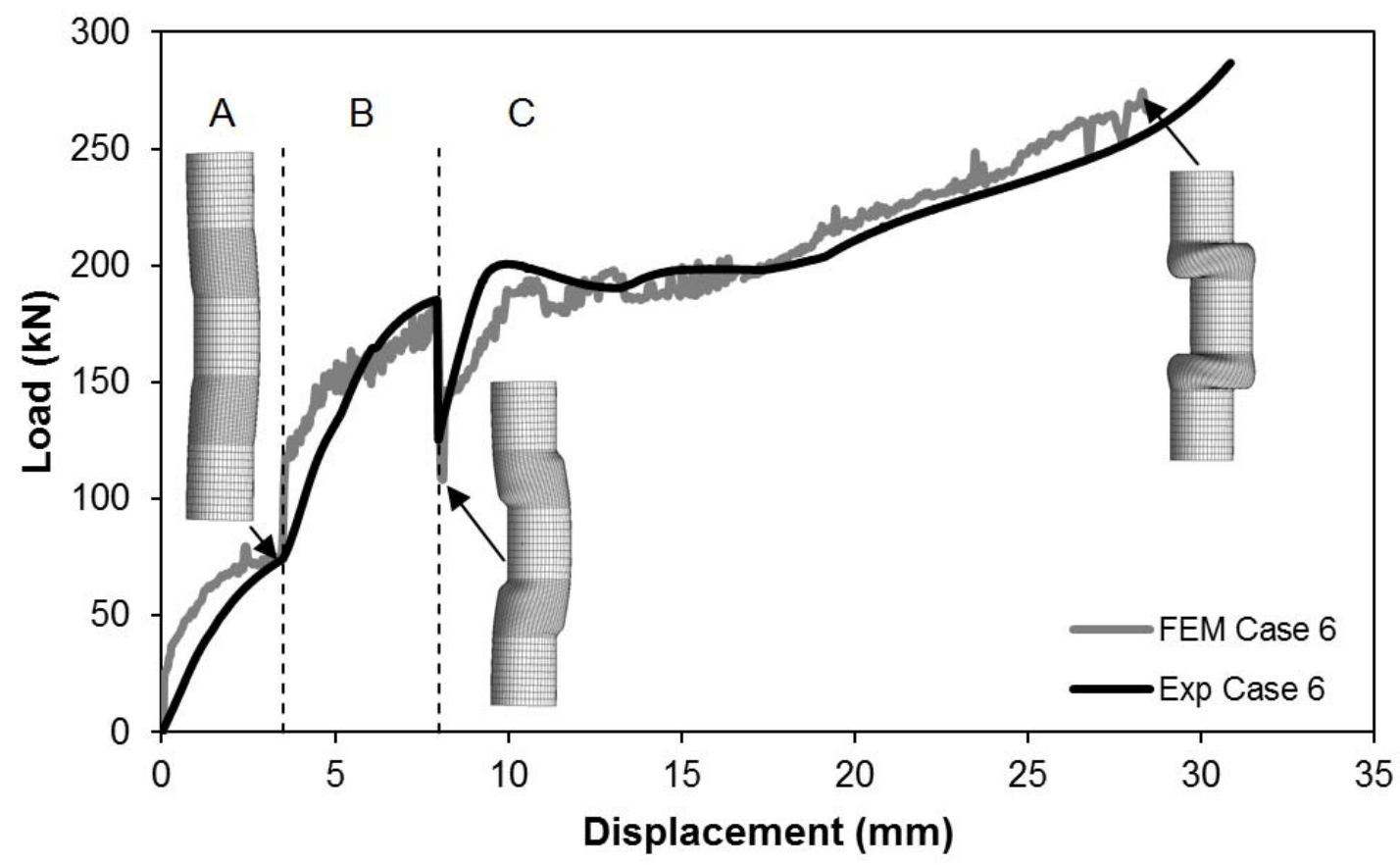

Figure 8 - Experimental and finite element predicted evolution of the load with displacement of the upper die shoe for the manufacture of tubular components with eccentric (gooseneck) cross sections by pressure-assisted forming with a solid lead medium (Case 6 of Table 1).

In region $\mathrm{A}$ the load increases monotonically towards a near steady-state value of approximately $70 \mathrm{kN}$ as a result of bending the carbon steel tube filled up with lead by means of pressure rings in order to prevent wall collapse and loss of circularity along the eccentric (gooseneck) cross sections. 
The sharp increase in the load up to $185 \mathrm{kN}$ in region $\mathrm{B}$ is attributed to plastic deformation being carried out by simultaneous action of the vertical and horizontal actuators in what may be considered as controlled buckling under axial compression loading and internal pressure.

The drop in load at the beginning of region $\mathrm{C}$ is caused by the removal of the vertical actuator (region C) and the subsequent drop in load rate is justified by the increase of eccentricity with buckling, which progressively diminishes the need to increase the axial compression load.

The experimental evolution of the load with displacement for Cases 4, 5 and 6 that is illustrated in Figure 9 allows establishing a relationship between the existence or nonexistence of the aforementioned regions $\mathrm{A}, \mathrm{B}$ and $\mathrm{C}$ and the movements of the actuators that are utilized in each case. In fact, Case 4 only allows identifying two different regions (similar to regions $\mathrm{A}$ and $\mathrm{C}$ of Case 6) whereas Case 5 only allows identifying a single region (similar to region B plus C of Case 6).

The evolution of the load with displacement for Case 1 is included as a reference and shows the result of collapse by buckling due to absence of pressurizing solid medium when combination of the vertical applied load and axial compression load exerted by the horizontal sliding dies reaches approximately $145 \mathrm{kN}$. 


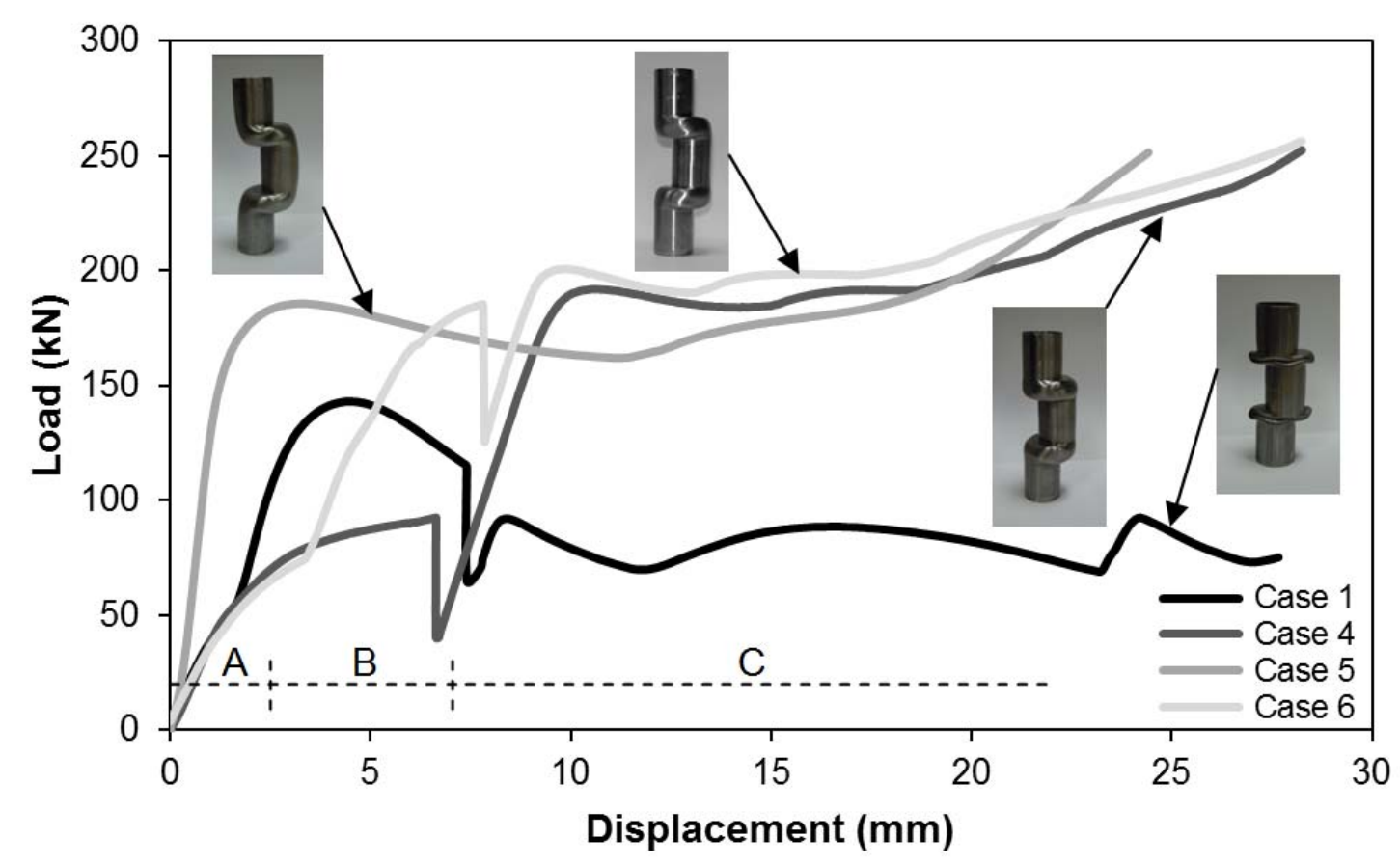

Figure 9 - Experimental evolution of the load with displacement of the upper die shoe for the manufacture of tubular components with eccentric (gooseneck) cross sections for Cases 1, 4, 5 and 6 of Table 1.

\section{CONCLUSIONS}

Pressure-assisted forming of carbon steel tubes using lead as a solid medium in a double-action tool system is a low-cost technological alternative to tube hydroforming in case of low-batch production of tubular components with eccentric (gooseneck) geometries.

The use of lead or any other low melting point metallic alloy as a solid medium has the advantage of easy removal from the tubular components after forming and recyclability. Adequate selection of the total amount of displacement $s$ in the direction perpendicular to the longitudinal axis the straight tubular specimens $s<2 r_{0}$ (where $r_{0}$ is the outer radius) is necessary to prevent excessive thinning and subsequent failure by fracture in opening mode I (by tension) in the outer tube wall of the eccentric (gooseneck) cross sections. 
The order of action of the vertical and horizontal actuators is very important for the overall success and quality of the tubular components. Best results are obtained with the utilization of three different actions applied in sequence; (i) preform bending by single action of the vertical actuator, (ii) controlled buckling under axial compression load by combined action of the vertical and horizontal actuators, (iii) finishing by buckling under axial compression load due to single action of the horizontal actuator.

Alternative solutions making use of just one or two different actions of the vertical and horizontal actuators may lead to failure or to fabrication of components with excessive curvature and thinning in the transition regions from the original to the eccentric, displaced, cross sections.

\section{ACKNOWLEDGEMENTS}

The authors would like to acknowledge the support provided by Fundação para a Ciência e a Tecnologia of Portugal under LAETA - UID/EMS/50022/2013 and by MCG - Mind for Metal, Carregado, Portugal. The work of Sara Gamboa is gratefully acknowledged. 


\section{REFERENCES}

1. Ogura T. and Ueda T. Liquid bulge forming, Metalworking Production 1968; 24: 73-81.

2. Al-Qureshi H.A. Comparison between the bulging of thin-walled tubes using rubber forming technique and hydraulic forming process, Sheet Metal Industries 1970; 47: 607-612.

3. Dohmann F. and Hartl Ch. Tube hydroforming - research and practical application, Journal of Materials Processing Technology 1997; 71: 174-186.

4. Ngaile G. Tube hydroforming, in Sheet Metal Forming - Processes and Applications (Editors: Altan T. and Tekkaya A.E.), ASM International 2012; 179209.

5. Lee M.G. Korkolis Y.P. and Kim J.H. Recent developments in hydroforming technology, Journal of Engineering Manufacture 2015; 229: 572-596.

6. Mori K., Patwari A.U. and Maki S. Improvement of formability by oscillation of internal pressure in pulsating hydroforming of tube, CIRP Annals of Manufacturing Technology 2004; 53: 215-218.

7. Xu Y., Zhang S.H., Cheng M. and Song H.W. Formability improvement of austenitic stainless steel by pulsating hydroforming, Journal of Engineering Manufacture 2015; 229: 609-615.

8. Yang C and Ngaile G. Preform design for tube hydroforming based on wrinkle formation, Journal of Manufacturing Science and Engineering - Transactions ASME 2011; 133: 061014.

9. Yang C., Li P., Hu Y. and Fan L. Reliability analysis of corner filling in the tube hydroforming process, Journal of Engineering Manufacture 2015; 229: 631-638 
10. Thiruvarudchelvan S. Elastomers in metal forming: A review, Journal of Materials Processing Technology 1993; 39: 55-82.

11. Ramezani M. and Ripin Z.M., Rubber pad forming processes. Woodhead Publishing, Cambridge, UK, 2012.

12. Girard A.C., Grenier Y.J. and Mac Donald B.J. Numerical simulation of axisymmetric tube bulging using a urethane rod, Journal of Materials Processing Technology 2006; 172: 346-355.

13. Alves L.M., Leitão P.M.F. and Martins P.A.F., Elastomer-assisted compression beading of tubes, Journal of Engineering Manufacture 2014; 228: 744-756.

14. Qin Y., Ma Y. and Balendra R. Pressurising materials and process design considerations of the pressure-assisted injection forging of thick-walled tubular components, Journal of Materials Processing Technology 2004; 150: 30-39

15. Alves L.M., Pardal T.C.D. and Martins P.A.F. Forming of thin-walled hollow spheres using sacrificial polymer mandrels, International Journal of Machine Tools and Manufacture 2009; 49: 521-529.

16. Mac Donald B.J. and Hashmi M.S.J. Three dimensional finite element simulation of bulge forming using a solid bulging medium, Finite Elements in Analysis and Design 2000; 37: 107-116.

17. Nielsen C.V., Zhang W., Alves L.M., Bay N. and Martins P.A.F. Modeling of Thermo-Electro-Mechanical Manufacturing Processes with Applications in Metal Forming and Resistance Welding. Springer-Verlag, London, UK, 2013.

18. Nielsen C.V., Zhang W., Martins P.A.F and Bay N. 3D numerical simulation of projection welding of square nuts to sheets, Journal of Materials Processing Technology 2015; 215: 171-180. 